Article

\title{
Simple Metal and Binary Alloy Phases Based on the $f c c$ Structure: Electronic Origin of Distortions, Superlattices and Vacancies
}

\author{
Valentina F. Degtyareva * and Nataliya S. Afonikova \\ Institute of Solid State Physics, Russian Academy of Sciences, Chernogolovka 142432, Russia; natasha@issp.ac.ru \\ * Correspondence: degtyar@issp.ac.ru; Tel.: +7-496-522-8376; Fax: +7-496-522-8160 \\ Academic Editor: Sławomir J. Grabowski \\ Received: 13 November 2016; Accepted: 23 January 2017; Published: 28 January 2017
}

\begin{abstract}
Crystal structures of simple metals and binary alloy phases based on the face-centered cubic $(f c c)$ structure are analyzed within the model of Fermi sphere-Brillouin zone interactions to understand the stability of the original cubic structure and derivative structures with distortions, superlattices and vacancies. Examination of the Brillouin-Jones configuration in relation to the nearly-free electron Fermi sphere for several representative phases reveals significance of the electron energy contribution to the phase stability. Representation of complex structures in the reciprocal space clarifies their relationship to the basic cubic cell.
\end{abstract}

Keywords: crystal structure; Hume-Rothery phases; structure stability

\section{Introduction}

Crystal structures of metals have three principal lattices: face-centered cubic ( $f c c)$, close-packed hexagonal $(h c p)$ and body-centered cubic $(b c c)$ with high symmetry and a close packing atomic arrangement. The prevalence of these structures is determined by high symmetry and high coordination numbers with the resulting high values of the Madelung constant and low electrostatic term of the crystal energy, called Ewald energy, $E_{\text {Ewald }}$. Another significant crystal energy term is the band structure energy, $\mathrm{E}_{\mathrm{BS}}$, defined by ion-electron interactions for a given structure.

Intermetallic compounds are mostly based on these types of structures with deformations, superlattices and vacancies, caused by differences in atomic sizes and electronegativities of components. One of the key factors for phase stability is "electron concentration"- - a number of valence electrons per atom [1-3]. Crystal energy is reduced due to the level of the valence electron energy (Fermi level) approaching the Brillouin zone plane and causing a formation of an energy gap.

The binary alloy system $\mathrm{Cu}-\mathrm{Zn}$ represents a classical example of Hume-Rothery phases that include the key metallic close-packed structures: $f c c, h c p$ and $b c c$. The sequence of the phases as a function of the average number of valence electrons per atom $f c c \rightarrow b c c \rightarrow$ complex $\gamma$-phase $\rightarrow h c p$ is defined by the following values of the valence electron concentration $1.35 \rightarrow 1.5 \rightarrow 1.62 \rightarrow 1.75$ (electron/atom). Similar phase sequences have been observed in many other binary alloy systems containing a noble metal from the group IB and a neighboring element from groups IIB-VB. The $\mathrm{Cu}-\mathrm{Zn}$ alloy system is a relatively simple system with only five intermediate phases that exhibit quite simple structures with the ground high-symmetry metallic phases like $f c c, b c c$ and $h c p$. Along with these basic metallic structures, there are phases $\mathrm{Cu}_{5} \mathrm{Zn}_{8}-c I 52$ and $\mathrm{CuZn}_{3}-h P 3$ that are related to $b c c$ through superlattices, distortions and vacancies. In our previous papers, we have considered metallic phases based on the $b c c$ and $h c p$ structures $[4,5]$.

Recently, we have considered intermediate phases in the $\mathrm{Au}-\mathrm{Cd}$ alloy system that is isoelectronic to the $\mathrm{Cu}-\mathrm{Zn}$ alloy system [6]. The Au-Cd phase diagram is more complicated containing about 
12 intermediate phases that are related to the basic high-symmetry structures as well as the structurally complex phases defined by Hume-Rothery rules. Formation of the larger number of the structures with many-faced $\mathrm{BZ}$ polyhedrons in the $\mathrm{Au}-\mathrm{Cd}$ phases comparing to the $\mathrm{Cu}-\mathrm{Zn}$ phases is stimulated by the higher atomic numbers and higher difference in atomic numbers of constituent elements.

In the present work, we analyze necessary conditions for stability of the $f c c$ structure and consider derivative complex structures. The subjects of analysis are the $s p$ metals and binary alloys for which it is possible to assign a definite number of valence electrons.

\section{Theoretical Background and Method of Analysis}

Formation of binary compounds at a certain alloy composition is defined by some important factors such as the difference in atomic sizes, electronegativity, etc. Beyond these factors, formation of metallic structures is defined by effects of the Fermi sphere-Brillouin zone (FS-BZ) interaction. The Hume-Rothery mechanism has been identified to play a role in the stability of structurally complex alloy phases, quasicrystals and their approximants [7-11]. The Jones model can be used to account for the phase stability in tetrahedral cluster structures, icosahedral and trigonal-prismatic clusters as building blocks. Formation of the complex structures of elemental metals under pressure can also be related to the Hume-Rothery mechanism [12-17].

The band structure contribution to the crystal structure energy can be estimated by analyzing configurations of Brillouin-Jones zone planes in the nearly free-electron model. A special program BRIZ has been developed [18] to construct FS-BZ configurations and to estimate some parameters such as the Fermi sphere radius $\left(\mathrm{k}_{\mathrm{F}}\right)$, values of reciprocal wave vectors of BZ planes $\left(\mathrm{q}_{\mathrm{hkl}}\right)$ and volumes of $\mathrm{BZ}$ and FS. The BZ planes are selected to match the condition $\mathrm{q}_{\mathrm{hkl}} \approx 2 \mathrm{k}_{\mathrm{F}}$ that have a significant structure factor. In this case, an energy gap is opened on the BZ plane leading to the lowering of the electron band energy. The ratio of $1 / 2 q_{\mathrm{hkl}}$ to $\mathrm{k}_{\mathrm{F}}$ is usually less than 1 and equals $\sim 0.95$; it is called a "truncation" factor. In the FS-BZ presentations by the BRIZ program, the BZ planes cross the FS, whereas in the real system, the Fermi sphere is deformed and accommodated inside BZ. The "truncation" factor has a characteristic value of $\sim 0.95$ and corresponds to a decrease in the electron energy on the BZ plane.

The crystal structure of a phase chosen for the analysis by the BRIZ program is characterized by the lattice parameters and the number of atoms in the unit cell, which define the average atomic volume $\left(V_{a t}\right)$. The valence electron concentration $(z)$ is the average number of valence electrons per atom that gives the value of the Fermi sphere radius $\mathrm{k}_{\mathrm{F}}=\left(3 \pi^{2} \mathrm{z} / \mathrm{V}_{\mathrm{at}}\right)^{1 / 3}$. Further structure characterization parameters are the number of BZ planes that are in contact with the FS, the degree of "truncation" factor and the value of BZ filling by electronic states, defined as a ratio of the volumes of FS and BZ.

Presentations of the FS-BZ configurations are given with the orthogonal axes with the following directions in the common view: $\mathrm{a}^{*}$ is looking forward, $\mathrm{b}^{*}$ - to the right and $\mathrm{c}^{*}$-upward. For the hexagonal lattice in the reciprocal space, $\mathrm{a}^{*}=\mathrm{a}_{1 \mathrm{~h}}{ }^{*} \cos 30^{\circ}, \mathrm{b}=\mathrm{a}_{2 \mathrm{~h}}{ }^{*}$ and $\mathrm{c}^{*}=\mathrm{c}_{\mathrm{h}}{ }^{*}$. Structural data for binary phases considered in this paper have been found in the Pauling File [19] and in recent papers cited in the corresponding sections of this paper.

\section{Results and Discussion}

In this work, we selected two groups of $f_{c c}$ based phases: elemental simple $s p$ metals (Table 1) and binary alloy phases (Table 2). Diffraction patterns and constructed Brillouin-Jones zones for these structures are presented in Figures 1 and 2, respectively. Crystal structure description and element groups in the Periodic table are given following Pearson [20]. 
Table 1. Structure parameters of several metal phases with the $f c c$ and $f c c$-based structures. Fermi sphere radius $\mathrm{k}_{\mathrm{F}}$, ratios of $\mathrm{k}_{\mathrm{F}}$ to distances of Brillouin zone planes $1 / 2 \mathrm{q}_{\mathrm{hkl}}$ and the filling degree of Brillouin zones (BZ) by electron states $V_{F S} / V_{B Z}$ are calculated using the program BRIZ [18].

\begin{tabular}{|c|c|c|c|c|c|c|}
\hline Phase & $\mathrm{Cu}(\mathrm{Zn})$ & $\alpha-\mathrm{Hg}$ & $\beta-\mathrm{Hg}$ & $\mathbf{L i}$ & In & $\mathrm{Te}$ \\
\hline Pearson symbol & $c F 4$ & $h R 1$ & $t 12$ & $h R 3$ & $t 12$ & $c F 4$ \\
\hline \multicolumn{7}{|c|}{ Structural data ${ }^{a}$} \\
\hline Space group & $F m \overline{3} m$ & $R \overline{3} m(h)$ & $\mathrm{I} / \mathrm{mmm}$ & $R \overline{3} m$ & $I 4 / \mathrm{mmm}$ & $F m \overline{3} m$ \\
\hline $\mathrm{T}, \mathrm{P}$ conditions & & $227 \mathrm{~K}$ & $77 \mathrm{~K}$ & $4.2 \mathrm{~K}$ & $\begin{array}{l}\text { Ambient } \\
\text { conditions }\end{array}$ & $255 \mathrm{GPa}$ \\
\hline lattice parameters $(\AA)$ & $a=3.698$ & $\begin{array}{c}a=3.470 \\
c=6.719 \\
c / a=1.936\end{array}$ & $\begin{array}{c}a=3.995 \\
c=2.825 \\
c / a=0.707\end{array}$ & $\begin{array}{l}a=3.111 \\
c=22.86\end{array}$ & $\begin{array}{c}a=3.248 \\
c=4.946 \\
c / a=1.523\end{array}$ & $a=3.757$ \\
\hline \multicolumn{7}{|c|}{ FS-BZ data from the BRIZ program } \\
\hline $\begin{array}{l}\mathrm{Z} \text { (number of valence } \\
\text { electrons per atom) }\end{array}$ & 1.364 & 2 & 2 & 1 & 3 & 6 \\
\hline $\mathrm{k}_{\mathrm{F}}\left(\AA^{-1}\right)$ & 1.473 & 1.364 & 1.380 & 1.116 & 1.504 & 2.375 \\
\hline $\begin{array}{l}\text { Total number } \\
\text { BZ planes }\end{array}$ & 14 & 14 & 12 & 26 & 14 & 12 \\
\hline $\begin{array}{l}\mathrm{k}_{\mathrm{F}} /\left(1 / 2 \mathrm{q}_{\mathrm{hkl}}\right) \\
\max \\
\min \end{array}$ & $\begin{array}{l}1.001 \\
0.867\end{array}$ & $\begin{array}{l}1.191 \\
0.972 \\
\end{array}$ & $\begin{array}{l}1.241 \\
1.013\end{array}$ & $\begin{array}{l}0.951 \\
0.825\end{array}$ & $\begin{array}{l}1.300 \\
1.099\end{array}$ & 1.049 \\
\hline$V_{F S} / V_{B Z}$ & 0.682 & 1.0 & 1.0 & 0.590 & 1.5 & 0.750 \\
\hline
\end{tabular}

\subsection{Simple sp Elements with the fcc-Based Structures}

The $f c c$ structure is one of the fundamental structures for metals along with $h c p$ and $b c c$ structures. These three atomic arrangements are characterized by high symmetry and close packing of atoms. It is interesting to consider chemical and physical factors that lead to the formation of complex and distorted phases-derivatives from these structures. Previously, we have considered simple metal structures based on $b c c$ and $h c p[4,5]$ demonstrating the valence electron concentration as a defining factor of structural stability.

\subsection{1. $f c c$ in the Simple sp Elements}

The group IB elements $\mathrm{Cu}, \mathrm{Ag}$ and $\mathrm{Au}$ crystallize in the $f_{c c}$ structure and do not change by variation of temperature or pressure, adhering to their name: "noble" metals. It should be noted that only for Au was the $f c c-h c p$ transition experimentally observed above 250 GPa [22].

The group IA elements $\mathrm{Li}$ and others adopt at ambient pressure the $b c c$ structure and transform to $f c c$ with the pressure increase [23]. The difference in the ambient pressure structure for univalent elements from IA and IB group can be explained by the balance of the electrostatic (Madelung) and the valence electron (band structure) terms in the crystal structure energy. Alkali IA elements adopt $b c c$ predominantly due to the electrostatic energy because the Madelung constant is higher for $b c c$ structure than for $f c c$ or $h c p$. Under pressure, the band structure term increases leading to the formation of $f c c$ for alkali metals.

The group IB elements follow the transition metals in the periodic table, and they have a filled $d$-electron band. Therefore, there is some screening of $E_{E w a l d}$ and gaining of $E_{B S}$. The common view of the Fermi surface for $\mathrm{Cu}, \mathrm{Ag}$ and $\mathrm{Au}$ is a sphere with necks extending to the Brillouin planes of the (111) type.

The $f c c$ structure remains stable for elements IB in the solid solution with the neighboring elements IIB and others. The limit of solubility is defined by the Hume-Rothery rule: at the valence electron 
concentration $\sim 1.36$, there is a contact of the Fermi sphere and the Brillouin Zone and the loss of stability for $f c c$. This case is shown for the $\mathrm{Cu}(\mathrm{Zn})$ alloy in Figure 1a.

a
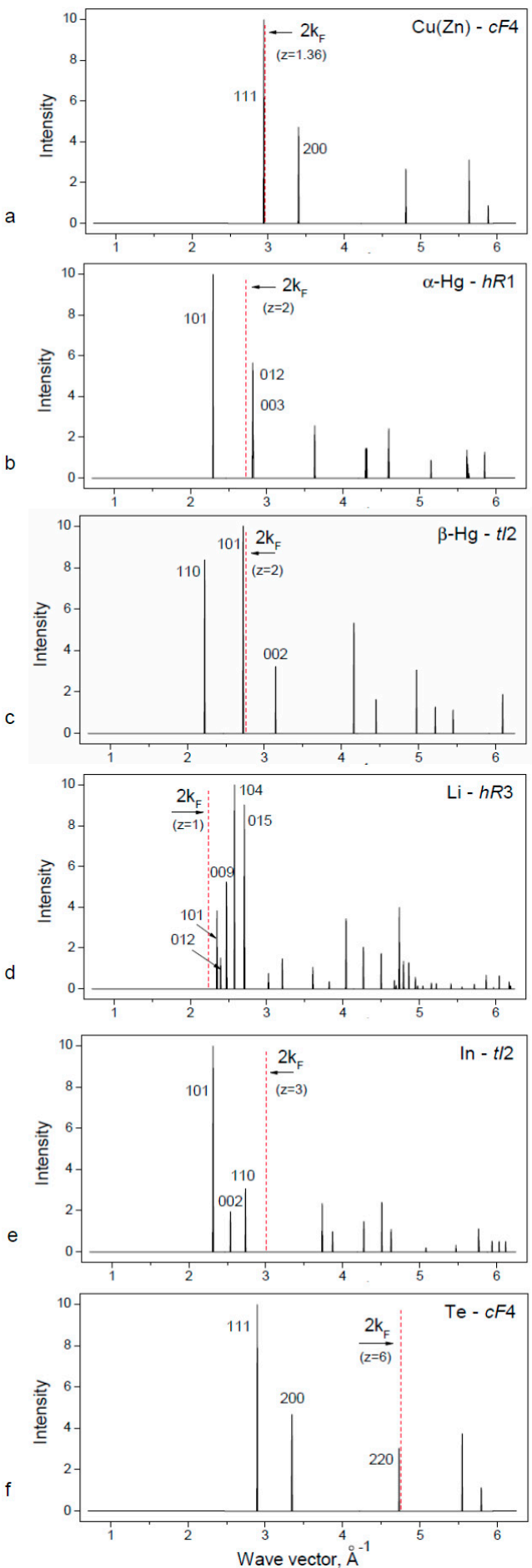
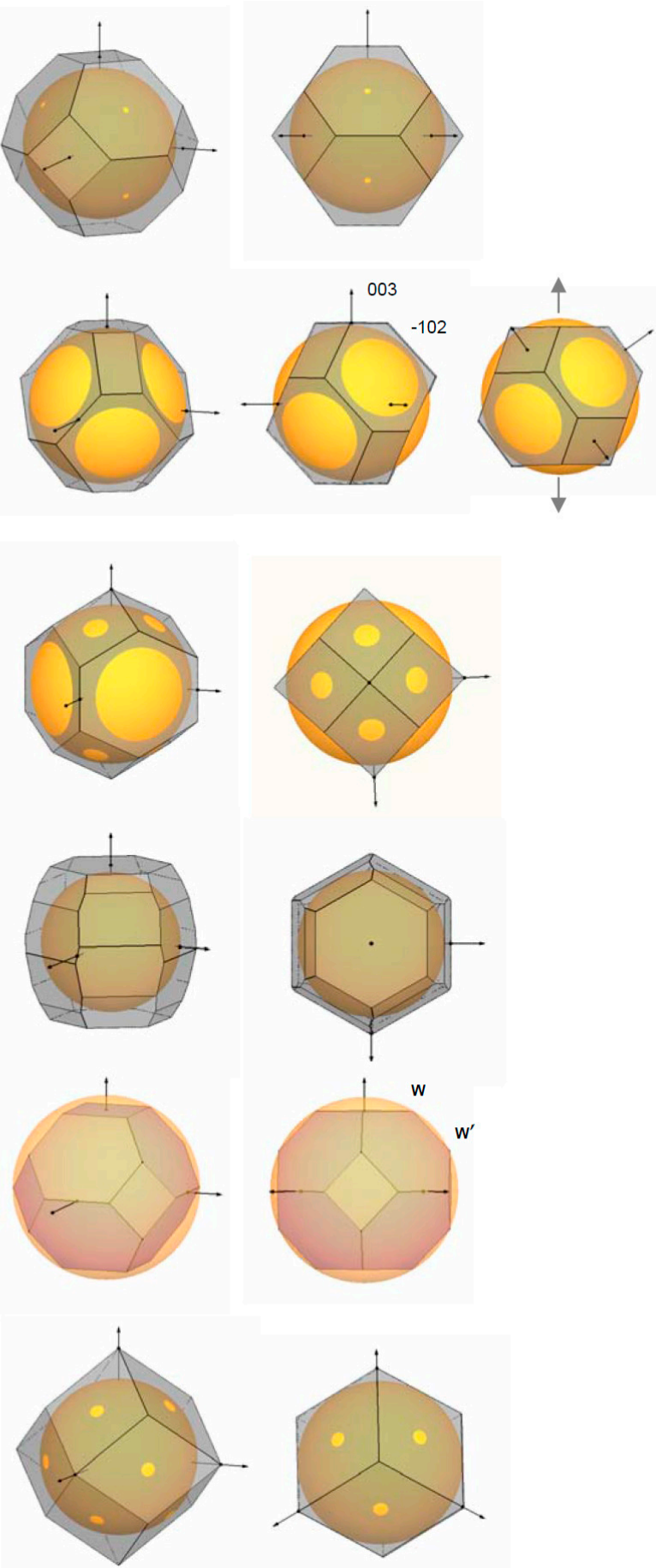

Figure 1. Calculated diffraction patterns for selected phases from Table 1 (left panel) and corresponding Brillouin-Jones (BZ) zones with the Fermi spheres (FS) (right panel). The position of $2 k_{F}$ and the $\mathrm{hkl}$ indices of the planes used for the $\mathrm{BZ}$ construction are indicated on the diffraction patterns. Constructions of BZ-FS are given in common projection (first column) and in special projection (second column) (see text). 


\subsubsection{Distorted fcc Structures of the IIB Element Hg}

The light group IIB elements $\mathrm{Zn}$ and Cd crystallize in the $h c p$ structures with the elongated $c / a$ ratio analyzed in [5]. The heavy IIB element $\mathrm{Hg}$ adopts distortive $f c c$ structures: $h R 1$ for $\alpha$-Hg and $t I 2$ for $\beta-\mathrm{Hg}$ (Table 1). The rhombohedral $\alpha-\mathrm{Hg}$ structure is closely related to $f c c$, as can be seen in Figure $1 \mathrm{~b}$ from the construction of Brillouin zones for $\mathrm{Hg}-h R 1$ and for the hypothetical $f c c$ with the same volume. BZ planes of the (111) type for $f c c$ are divided into two sets of $h R 1$ of (101) and (003) types in such a way that the latter is placed outside FS at the same distance as (012) planes, which are the former (200)-fcc planes. Rhombohedral distortion degree is defined by the condition $\mathbf{q}_{003}=\mathbf{q}_{012}$ resulting in $c / a=1.936$ for the rhombohedral cell in the hexagonal setting. This value is much lower than the ideal $c / a=2.45$ for $f c c . H g-h R 1$ is obtained from $f c c$ through contraction along one of the [111] axes as shown on Figure $2 \mathrm{~b}$ (right panel). Rhombohedral cell of the $\mathrm{Hg}-h \mathrm{R} 1$ structure has $\alpha=70.52^{\circ}$ in comparison to $60^{\circ}$ of the $f c c$. At low temperature and high pressure, the $\beta-\mathrm{Hg}$ phase is stable.

The $\beta-\mathrm{Hg}$ structure can be described as a strong compression of $f_{c c}$ along the $c$-axis to $c / a \sim 0.5$. Standard description of $\beta-\mathrm{Hg}$ is as a body-centered tetragonal structure, $b c t$, with $c / a \sim 0.707$ (close to $1 / \sqrt{2}$ ). For this structure, (101) planes are in contact with the FS as shown in Figure 2c. The coordination number for $\mathrm{Hg}$ - $b c t$ is close to $\mathrm{N}=10$.

The alloying of $\mathrm{Hg}$ with $\mathrm{Sn}$ under pressure was studied in [24]. The solubility of $\mathrm{Sn}$ in $\alpha-\mathrm{Hg}$ was found to increase under pressure up to $\sim 10$ at. $\% \mathrm{Sn}$. The qualitative estimation from the condition $\mathrm{k}_{\mathrm{F}}$ $\sim 1 / 2 q_{012}$ gives the value $\mathrm{z} \sim 2.17$, which corresponds to $\sim 8-9$ at. $\%$ of a tetravalent metal and to $\sim 17$ at. $\%$ of a trivalent metal. The latter case is realized in the $\mathrm{Hg}-\mathrm{In}$ system, where the $\alpha-\mathrm{Hg}(\mathrm{In})$ solution has been experimentally observed to extend to $\sim 19$ at. \% In. Stability of the $\alpha-\mathrm{Hg}(\mathrm{Sn})$ phase was observed to $30 \mathrm{GPa}$.

For pure $\mathrm{Hg}$, transformation $\alpha-\beta$ occurs under pressure of $\sim 3.4 \mathrm{GPa}$ at room temperature with further transitions at $12 \mathrm{GPa}$ to monoclinic structure $(\gamma)$ and at $37 \mathrm{GPa}$ to the close-packed hexagonal $(h c p)$ structure [25,26]. Theoretical calculation [27] has shown that the $f c c$ structure is extremely close to $\beta$-and $\gamma-\mathrm{Hg}$. It should be noted that, for IIB elements, $\mathrm{Zn}, \mathrm{Cd}$ and $\mathrm{Hg}$, the common $h c p$ phase exists under high pressure. The $\beta-\mathrm{Hg}$ phase is stabilized at ambient pressure in the $\mathrm{Hg}-\mathrm{Cd}$ alloys at 4 to 70 at. \% Cd [19]. This occurrence may be accounted for by the chemical pressure because the atomic volume of $\mathrm{Cd}$ is less than that for $\mathrm{Hg}$ (21.6 and $23.2 \AA^{3}$, respectively).

\subsubsection{Rhombohedral Distortions of $f_{c c}$ in Group IA Element Li}

Rhombohedral structure of $\alpha-\mathrm{Hg}-h R 1$ type occurs in alkali element $\mathrm{Li}$ under pressure above $39 \mathrm{GPa}$ after the transition $f c c \rightarrow h R 1$ [28]. Lattice parameter of Li- $h R 1$ at $39.8 \mathrm{GPa}$ are $a=2.4023 \AA$, $c=5.516 \AA, c / a=2.296$ for the hexagonal cell and the rhombohedral angle is $62.87^{\circ}$. The degree of rhombohedral distortion of $f c c$ is less than for $\alpha-\mathrm{Hg}$. With this deformation, one set of the (111) planes of $\mathrm{BZ}$ for $f_{c c}$ is placed closer to the FS corresponding to $\mathrm{z}=1$, and there is some gain for the band structure energy term at the $f c c \rightarrow h R 1$ transition.

Another rhombohedral structure is known for $\mathrm{Li}$ and $\mathrm{Na}$ at low temperature at ambient pressure below $77 \mathrm{~K}$ and $25 \mathrm{~K}$, respectively. This structure, usually defined as 9R or Sm-type, is close-packed structure with the sequence of hexagonal layers $A B A B C B C A C A$ in contrast to the $A B A B$ sequence for $h c p$ and $\mathrm{ABCABC}$ for $f c c$. The physical source for energetic stability of the $9 \mathrm{R}$ phase was analyzed by Ashcroft using a Hume-Rothery argument [29]. The Brillouin-Jones zone of the 9R phase contains 26 planes that are close to the FS so that the overall electronic energy is reduced (see Table 1 and Figure $1 \mathrm{~d}$ ). The BZ for Li- $h R 3$ is filled by electronic states up to 0.59 that is slightly more than 0.5 for $\mathrm{Li}-b c c$.

\subsubsection{Distortion of $f c c$ in Group IIIB Elements}

The lightest trivalent element $\mathrm{Al}$ has $f c c$ structure and is found to be stable with the variation of temperature and pressure. This indicates the importance of the electrostatic (Madelung) contribution into 
the crystal energy for this light element. Recently at very high pressure of $217 \mathrm{GPa}$, the transformation $f c c \rightarrow h c p$ was observed for Al. For the heaviest IIIB metal thallium, the valence electron contribution is significant and the resulting ambient pressure structure is $h c p$. Under pressure $3.7 \mathrm{GPa}$, $\mathrm{Tl}$ transforms to $f c c$ [30] and remains $f c c$ up to $125 \mathrm{GPa}$ [31].

The intermediate group IIIB metal In crystallizes at ambient pressure in a tetragonal structure that is a slight distortion of $f c c$ with an elongation of $c$-axis to $c / a=1.076$. Standard description of In structure is the body centered tetragonal, $b c t$ or $t I 2$, as given in Table 1.

Physical reasons of the distorted In structure were considered within the model of Fermi sphere-Brillouin zone interactions [32]. The BZ polyhedron of $f c c$ is deformed due to the attraction to the FS, in particular at the one set of corners of $\mathrm{W}^{\prime}$ type. Dependence of $f c c$ distortion is defined by the valence electron concentration and the ideal $f c c$ is realized when the Fermi sphere contacts the BZ at the corners of $\mathrm{W}$ type: this case occurs for $\mathrm{z}=2.94$ electron/atom and is realized in the In alloys with $\mathrm{Cd}$ or $\mathrm{Hg}$ at about $\sim 6$ at. \%. The structural distortion of $f c c$ in In increases with the addition of 4-valent metals $\mathrm{Sn}$ or $\mathrm{Pb}$ up to $12-15$ at. \%. At higher electron concentration, there is a phase transition to the $f c t$ with $c / a<1$. The origin of existence of these two types of $f c t$ with $c / a>1$ and $c / a<1$ is related to different FS-BZ configurations where the FS is touched by the corners of BZ of $\mathrm{W}^{\prime}$ or $\mathrm{W}$ type, as discussed in $[13,32]$.

Similar tetragonal phase is formed in Ga-the lighter neighbor of In in IIIB group-under high pressure above $14 \mathrm{GPa}$ with $c / a \sim 1.12$ (for $f c t$ ). The Ga-fct phase has graduate transition to $f c c$, observed near $70 \mathrm{GPa}[23,33]$. Thus, for group III elements, the most abundant structure under compression is $f c c$ or slightly distorted $f c c$ structure.

\subsubsection{High Pressure $f c c$ Phase in Polyvalent sp Elements}

The group VIB elements contain six sp electrons, and their metallic phases obtained under pressure should be considered with respect not only to the first Brillouin zone but taking into account the planes of the larger Brillouin zones. An interesting case of the $f c c$ structure was found recently for the group VIB element Te [34]. At normal pressure, Te is non-metallic and its structure consists of a spiral chain (trigonal $h R 1$ ), which transforms under pressure to some complex structures including an incommensurately modulated structure [23] with metallic properties. Above $27 \mathrm{GPa}$, Te adopts the $b c c$ structure. Above $100 \mathrm{GPa}$, it transforms to $f c c$ with a superlattice and above $200 \mathrm{GPa}$ to the $f c c$ structure [34]. Consideration of Te- $f c c$ phase with $z=6$ shows the satisfaction with the FS-BZ interaction model (Table 1, Figure 1f). The FS contact with the BZ planes of the (220) type occurs for $z=5.92$, and, with $z=6$, there is a slight overlap with the FS near the $1 / 2 q_{220}$, which is usual for the Hume-Rothery phases.

Iodine an element from group VIIB — and a neighbor to TE-is a typical diatomic molecular solid under ambient conditions. Transition to the metallic state has been found for Iodine above $16 \mathrm{GPa}$ and several phases have been identified on the way from molecular to monatomic states including incommensurately modulated structure [35]. At pressures above $55 \mathrm{GPa}$, Iodine was found to crystallize in the $f c c$ structure [36], which is stable to at least $273 \mathrm{GPa}$ [37].

Appearance and stability of the $f c c$ structure in the elements of groups VIB and VIIB at high pressures is supported by the model of the FS-BZ interaction with consideration of the extended Brillouin-Jones zones for polyvalent metals. In particular, for $z=6$ and $z=7$, the energetic gain is obtained due to the accommodation of the FS electrons into the BZ formed by (220) planes.

It should be noted that, for group IVB elements, the $f c c$ appears only in the heaviest element $\mathrm{Pb}$ at normal pressure and transforms under compression to $h c p$ and then to $b c c$. For group VB elements, the common high pressure phase is $b c c$ (see reviews $[13,23]$ ).

\subsection{Binary Alloy Phases with the fcc Based Structures}

For the binary alloys, important factors of the phase formation are atomic size ratio and electronegativity. For our consideration, we select group IB elements with the neighboring group 
elements with the minimal differences of these two factors that are, however, sufficient for site-ordered phase formation. In all cases, it should be noted that the decisive factor for the structural stability is also the Hume-Rothery mechanism as is demonstrated for several representatives of binary phases (Table 2, Figure 2).
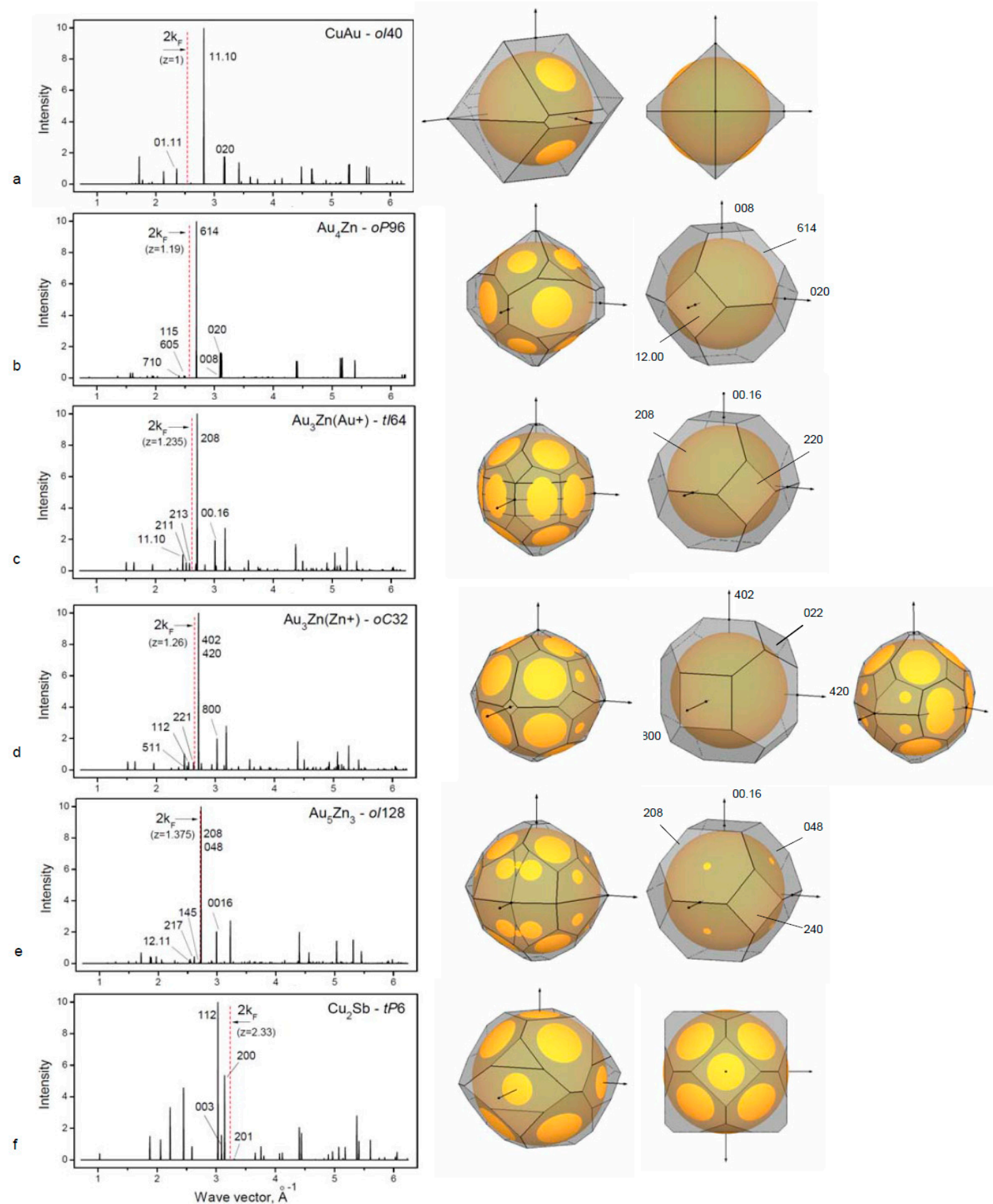

Figure 2. Calculated diffraction patterns for selected phases from Table 2 (left panel) and corresponding Brillouin-Jones zones with the inscribed Fermi spheres (right panel). The position of $2 k_{F}$ and the hkl indices of the planes used for the BZ construction (first column) are indicated on the diffraction patterns. Constructions of BZ planes corresponding to the strong reflections (second column) show structural relationship to the basic cells (see text). For BZ of $o C 32$ (d, third column) the projection is given along $\mathbf{b}^{*}$ to show relation to $o P 128$ (e). (See text for description of (a-f) sub-figures). 
Table 2. Structure parameters of several binary fcc-based phases from the literature data. Fermi sphere radius $\mathrm{k}_{\mathrm{F}}$, ratios of $\mathrm{k}_{\mathrm{F}}$ to distances of Brillouin zone planes $1 / 2 \mathrm{q}_{\mathrm{hkl}}$ and the filling degree of Brillouin zones by electron states $V_{F S} / V_{B Z}$ are calculated using the program BRIZ [18].

\begin{tabular}{|c|c|c|c|c|c|c|}
\hline Phase & $\mathrm{CuAu}$ & $\mathrm{Au}_{4} \mathrm{Zn}$ & $\mathrm{Au}_{3} \mathrm{Zn}(\mathrm{Au}+)$ & $\mathrm{Au}_{3} \mathrm{Zn}(\mathrm{Zn}+)$ & $\mathrm{Au}_{5} \mathrm{Zn}_{3}$ & $\mathrm{Cu}_{2} \mathrm{Sb}$ \\
\hline Pearson symbol & oI40 & oP96 & tI64 & $o C 32$ & $o I 128$ & $t P 6$ \\
\hline \multicolumn{7}{|c|}{ Structural data ${ }^{a}$} \\
\hline Space group & Imma & Pnmn & $I 4_{1} / \mathrm{acd}$ & Cmca & Ibam & $\mathrm{P} 4 / \mathrm{nmm}$ \\
\hline $\mathrm{T}, \mathrm{P}$ conditions & & & & $\begin{array}{l}\text { Ambient } \\
\text { conditions }\end{array}$ & & \\
\hline lattice parameters $(\AA)$ & $\begin{array}{l}a=3.676 \\
b=3.956 \\
c=39.72\end{array}$ & $\begin{array}{c}a=24.216 \\
b=4.025 \\
c=16.244\end{array}$ & $\begin{array}{l}a=5.586 \\
c=33.40\end{array}$ & $\begin{aligned} a & =16.603 \\
b & =5.581 \\
c & =5.581\end{aligned}$ & $\begin{array}{c}a=5.510 \\
b=11.020 \\
c=33.620\end{array}$ & $\begin{array}{l}a=4.001 \\
c=6.104\end{array}$ \\
\hline \multicolumn{7}{|c|}{ FS-BZ data from the BRIZ program } \\
\hline $\begin{array}{l}\mathrm{Z} \text { (number of valence } \\
\text { electrons per atom) }\end{array}$ & 1 & 1.19 & 1.235 & 1.26 & 1.375 & 2.33 \\
\hline $\mathrm{k}_{\mathrm{F}}\left(\AA^{-1}\right)$ & 1.27 & 1.288 & 1.310 & 1.322 & 1.367 & 1.618 \\
\hline $\begin{array}{l}\text { Total number } \\
\text { BZ planes }\end{array}$ & 14 & 28 & 50 & 34 & 34 & 22 \\
\hline $\begin{array}{l}\mathrm{k}_{\mathrm{F}} /\left(1 / 2 \mathrm{q}_{\mathrm{hkl}}\right) \\
\max \\
\min \end{array}$ & $\begin{array}{l}1.0078 \\
0.7996\end{array}$ & $\begin{array}{l}1.0756 \\
0.825\end{array}$ & $\begin{array}{l}1.0635 \\
0.8705\end{array}$ & $\begin{array}{l}1.069 \\
0.873\end{array}$ & $\begin{array}{l}1.046 \\
0.914\end{array}$ & $\begin{array}{l}1.0686 \\
0.979\end{array}$ \\
\hline$V_{F S} / V_{B Z}$ & 0.600 & 0.875 & 0.972 & 0.968 & 0.921 & 0.916 \\
\hline
\end{tabular}

\subsubsection{Long-Period Superlattice in the CuAu Alloy}

$\mathrm{Cu}$ and $\mathrm{Au}$ are both univalent group IB metals with the atomic size difference $\sim 12 \%$. The $\mathrm{CuAu}$ alloy forms the tetragonally distorted $f c c$ lattice where alternate (00h) planes contain either $\mathrm{Cu}$ or $\mathrm{Au}$ atoms and cause a contraction in $c$ direction. Resulting tetragonal face-centered structure has $c / a$ ratio of 0.92 [19]. In the temperature range $\sim 380^{\circ} \mathrm{C}$ to $410{ }^{\circ} \mathrm{C}$, the superlattice $\mathrm{CuAuII}$ is formed which consist of $\mathrm{CuAu}$-bct unit cells with the antiphase domains along the $b$-direction. There is a lattice shift of $1 / 2(a+c)$ at each five unit-cell length $[38,39]$. The superlattice $\mathrm{CuAu}$ II is described as orthorhombic cell with 10 cells along one of a direction, oI 40 .

The stabilization of long-period superlattices in $\mathrm{CuAu}$ and other alloys has been understood by considering that the Fermi surface touches the newly formed superlattice Brillouin zone boundaries $\{110\}$ that are adjusted due to the formation of an extra period. Figure $2 \mathrm{a}$ is showing the touching of the Fermi sphere to the planes (01.11). The degree of overlapping $\left(1 / 2 q_{h k l}\right) / k_{F}$ for ideal FS is usually $\sim 0.95$ as was considered by Sato and Toth [38] and is called a "truncation" factor. In our paper, we use the reciprocal value to characterize the FS-BZ interaction in all considered cases.

\subsubsection{Binary Alloy Au-Zn Phases Based on the $f c c$ Structure}

The $\mathrm{Cu}(\mathrm{Zn}) f c c$ solid solution (considered above in the Section 3.1.1) exists up to the electron concentration $\mathrm{z}=1.36$ without any ordered phases at low temperatures. For alloys with $\mathrm{Au}(\mathrm{Zn})$, the $f c c$ structure exists at high temperature $\left(403-683^{\circ} \mathrm{C}\right)$ within the same region of the electron concentration (up to $33 \% \mathrm{Zn}$ ). However, a number of ordered phases with long-period superlattices were found at low temperature [19]. Single crystal studies of 19, 23.5 and 26 at. \% Zn by X-ray diffraction [40,41] allow structure characterization of these phases. We consider possible mechanism of structural stability due to formation of additional Brillouin zone plane causing lattice modulations (Table 2, Figure $2 \mathrm{~b}-\mathrm{e}$ ).

For the $\mathrm{Au}_{4} \mathrm{Zn}$-oP96 structure, the superlattice cell is related to the basic $f c c$ cell (with $a_{\mathrm{f}}$ parameter) with a model: $a=6 a_{\mathrm{f}}, b=a_{\mathrm{f}}$ and $c=4 a_{\mathrm{f}}$ [32]. Atomic positions are varied corresponding to the lattice modulation. In Figure $2 b$, the new BZ boundaries of the (115) and (710) types are shown crossing the FS, whereas for the basic BZ (shown right), there is no contact with FS. The phase stabilization is 
considered to be due to the formation of the new BZ planes in contact with the FS, which is reducing the crystal energy.

$\mathrm{Au}_{3+} \mathrm{Zn}$-tI64 phase is related to $f c c$ in the following way: $a=\sqrt{2} a_{f}, c=8 a_{f}$. Atomic shifts produce extra diffraction reflections [42], resulting in the BZ boundaries enveloping the FS (Figure 2c).

$\mathrm{Au}_{3} \mathrm{Zu}_{+}-o C 34$ phase occurs through a phase transition from $f c c$ in a similar way with $b$ and $c$ $\sim \sqrt{2} a_{f}$ and $a=4 a_{\mathrm{f}}$ (standardized data are used). FS-BZ construction is given in Figure $2 \mathrm{~d}$ showing relation of two $\mathrm{Au}_{3} \mathrm{Zn}$ phases with slight difference in composition.

$\mathrm{Au}_{5} \mathrm{Zn}_{3}-\mathrm{oI} 128$ phase has also a relation to $f c c$ and occurs at the alloy concentration boundary for the $f c c$ stability. Cell parameters are produced from $f c c$ cell in the following way: $a=\sqrt{2} a_{f}, b=2 \sqrt{2} a_{f}$ and $c=8 a_{\mathrm{f}}$, resulting in $32 \mathrm{fcc}$ cells with 128 atoms (Figure 2e). Thus, in the $\mathrm{Au}-\mathrm{Zn}$ alloy system in the composition range up to $\sim 40$ at. $\% \mathrm{Zn}$, there are several complex phases that are superlattices based on $f c c$. Formation of these superstructures is related to the Hume-Rothery mechanism.

\subsubsection{Defect Structures Based on $f c c$}

In this section, we consider another way of the structure adjustment to the Hume-Rothery rules via formation of defect structures with vacancies. For $\gamma$-brass in $\mathrm{Cu}-\mathrm{Ga}$ and $\beta$-(NiAl), it was found that with increasing the average number of valence electrons $z$, these structures can be stabilized by skipping atoms out of the unit cell. Thus, for stability of the phase, it is fundamental to keep the constant number of electrons per unit cell zN ( $\mathrm{N}$ is the atom number in the unit cell). In the $\mathrm{Cu}-\mathrm{Ga}$ alloy systems, the $\gamma$-brass exists in the wide range of compositions $30-43$ at. $\% \mathrm{Ga}$, and the number of atoms in the unit cell was found to decreased from 52 to 47 atoms [43].

A special case of defect superstructure is vacancies at certain atomic positions in the cell. The structure $\mathrm{Cu}_{2} \mathrm{Sb}-\mathrm{tP} 6$ is related to the tetragonal distortion of the $f c c$ with the double cell along the $c$-axis and with the absence of $\mathrm{Cu}$ atoms in the middle of $c$. The resulting number of atoms in the cell is $\mathrm{N}=6$ and the constructed FS-BZ configuration satisfies the Hume-Rothery mechanism (Figure 2f). Similar $t I 6$ structure is found in $\mathrm{Cu}_{2} \mathrm{As}$.

It is remarkable that the $\mathrm{Cu}_{2} \mathrm{Sb}-t P 6$ type structure is formed in the $\mathrm{Cu}$-Te alloy at the composition of 41.7 at. \% Te [19]. Because Te is an element from the next group (VIB) after the group of Sb (V B), there are more vacancies of $\mathrm{Cu}$ atoms for basic $\mathrm{Cu}$ positions $2 \mathrm{c}$ and 2a with occupation 0.7 , which gives the resulting number of atoms $\mathrm{N}=4.8$ in the unit cell instead of $\mathrm{N}=6$ for $\mathrm{Cu}_{2} \mathrm{Sb}$. This is the response of the crystal structure to FS-BZ interactions for the phase $\mathrm{Cu}_{1.4} \mathrm{Te}$.

Another example of defect supercell formation based on $b c c$ is $\mathrm{CuGa}_{2}-t P 3$ with two $b c c$ cells along the $c$-axis and with missing $\mathrm{Cu}$ atoms in the middle of $c$. This is a way to adjust structure to the number of valence electrons in the cell.

\subsection{Miscibility Gap of Two fcc Phases in the Al-Zn Alloys}

For polyvalent metals, the volume of the valence electrons, defined by the volume of the Fermi sphere, is always larger than the volume of the BZ. Therefore, the Fermi surface cuts many zone planes, and contributions to the crystal energy from regions near the plane intersections may lead to important effects for phase stability [44].

An interesting case of the $f_{c c}$ phase decomposition is observed in the Al- $\mathrm{Zn}$ alloy system, where $\mathrm{Al}(\mathrm{Zn}) f c c$ solid solution is stable at high temperatures, and, below $352^{\circ} \mathrm{C}$, there is a miscibility gap of two fcc phases with 16.5 and 59 at. \% $\mathrm{Zn}$. The atomic radii of $\mathrm{Al}$ and $\mathrm{Zn}$ are very close $(1.43 \AA$ and $1.37 \AA$, respectively) and electronegativities are nearly equal (1.61 and 1.65), so there must be some other factor that defines the decomposition [20]. In this case, it is the electronic structure factor (see Figure 3). Theoretical consideration of the electronic topology in the $f c c \mathrm{Al}-\mathrm{Zn}$ alloys has been performed in [45]. 


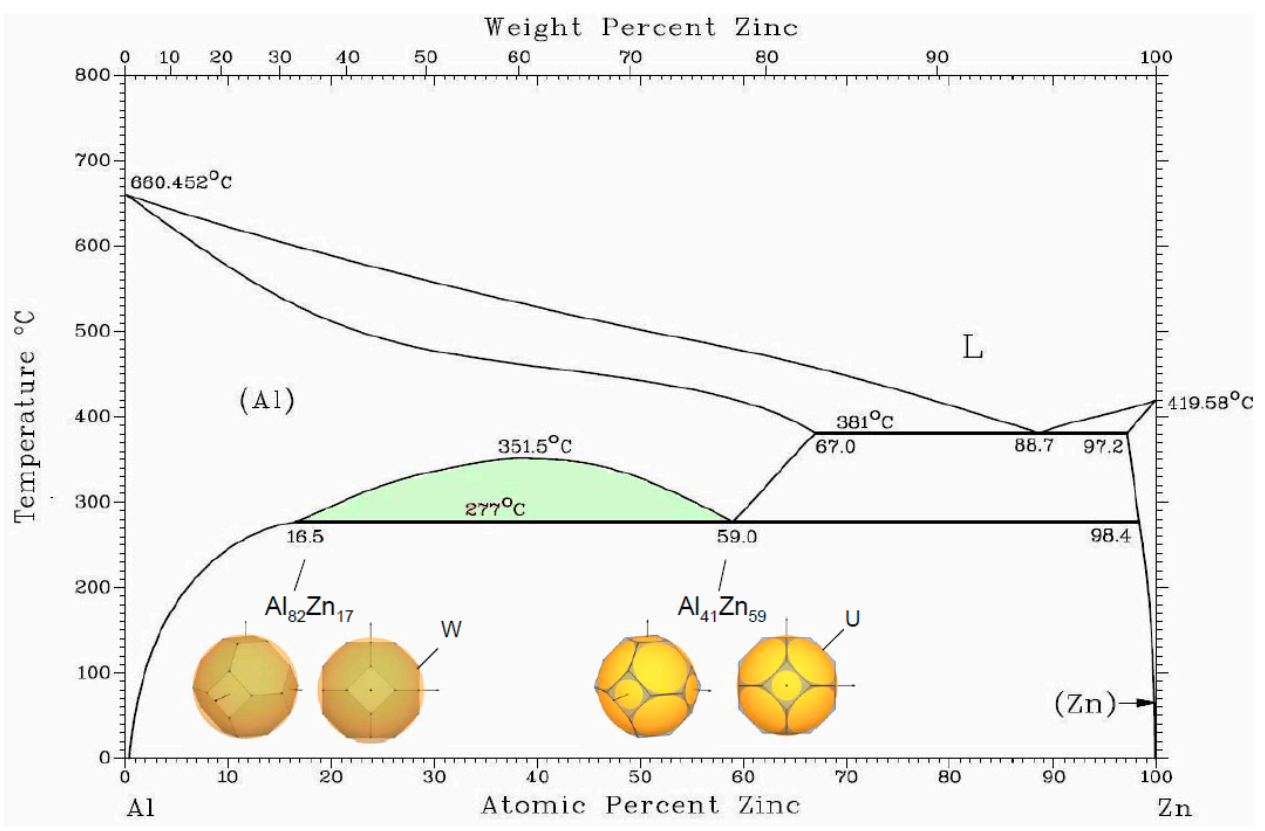

Figure 3. The phase diagram $\mathrm{Al}-\mathrm{Zn}[19]$. The $\mathrm{Al}(\mathrm{Zn})$ solid solution region with the $f c c$ structure contains a miscibility gap with two-phase region (colored). The boundary phases with 17 and 59 at. \% Zn constructions of FS-BZ are given with a common view and a view along a* (see text).

We consider coexistence of two $f c c \mathrm{Al}(\mathrm{Zn})$ phases with the configuration of FS and BZ. It was discussed for In- $f c c$ phase that the important factor for the structure stability is BZ accommodation within the Fermi sphere. The contact of FS with $\mathrm{W}$-type corners occurs for $\mathrm{z}=2.94$, which corresponds to addition of $\sim 7 \% \mathrm{Zn}$, and, due to the "truncation" factor, can be extended to $\mathrm{z}=2.83$, which corresponds to $\sim 17$ at. $\% \mathrm{Zn}$ as observed in one of the $f c c \mathrm{Al}-\mathrm{Zn}$ phase. Another factor for gaining in energy for $f c c$ is a contact to the edges of (111) planes in the points $\mathrm{K}$ and $\mathrm{U}$ of BZ that occurs at $\mathrm{z}=2.5$ for $\sim 50$ at. $\% \mathrm{Zn}$. This results in the "truncation" factor 1.036-very acceptable for the Hume-Rothery conditions. It should be noted that the region of miscibility gap of the two $\mathrm{Al}(\mathrm{Zn}) f_{c c}$ extends under pressure along the temperature [46] in accordance with the common trend that an energy gap increases under pressure.

\section{Conclusions}

The phase sequence in the $\mathrm{Cu}-\mathrm{Zn}$ alloy system consists of simple high-symmetry metallic structures $f c c, b c c$ and $h c p(\alpha, \beta$ and $\varepsilon$ ) with an addition of two phases $(\gamma-c I 52$ and $\delta-h P 3)$ that are derivatives of the $b c c$ structure. Phases $\alpha, \beta$ and $\varepsilon$ are completely site-disordered because $C u$ and $\mathrm{Zn}$, the constituent elements of the alloy, are close neighbors in the periodic table and have minimal differences in the atomic size and electronegativity. In the $\mathrm{Au}-\mathrm{Zn}$ alloy system in the composition range up to $\sim 40$ at. $\% \mathrm{Zn}$, there are several complex phases that are superlattices based on $f c c$. Formation of these superstructures is related to the Hume-Rothery mechanism. Additional complexity of the Au-Zn alloys in comparison to the $\mathrm{Cu}-\mathrm{Zn}$ alloys is related to the larger difference in atomic numbers of the alloy components that provides relatively strong diffraction peaks for site-ordered compounds. Remarkable example is a long-period superlattice of the $\mathrm{CuAu}$ with the size of domains defined by formation of superstructure reflections in contact to the Fermi sphere [38].

In the pure simple $s p$ elements, the $f c c$ phase is stable in group IB metals $(\mathrm{Cu}, \mathrm{Ag}, \mathrm{Au})$ and their solid solutions up to $\sim 1.35$ electron/atom. In this case, the FS is inside the first BZ and touched the (111) planes. For the group IIIB element In, the $f c c$ structure shows a tetragonal distortion that can be understood through the BZ deformation: FS accommodates the BZ by touching one type of the W corners. Similar tetragonal face-centered structure is observed for compressed Ga. The contact 
of the FS with the special points of BZ such as W-type corners or the edges of (111) planes of BZ in the points $\mathrm{K}$ and $\mathrm{U}$ can lead to a miscibility gap between two $f c c$ phases as in the case of Al-Zn alloys.

In conclusion of this discussion, it should be noted that the $f c c$ phase appears under pressure for group IA (alkali metals) and is followed by complex structures that show a decrease in coordination number and packing density (for $\mathrm{Li}$ and $\mathrm{Na}$ after $\mathrm{cl16}$, for $\mathrm{Rb}$ and $\mathrm{Cs}$ after oC52 and oC84, respectively) $[13,23]$. The reason for this unexpected structural complexity in alkali elements under strong compression can be understood if one accepts the change in the valence electron number due to the overlap of the valence band and upper core electron levels [14,15]. Thus, the application of the FS-BZ interaction model allows us to estimate the effective number of valence electrons in the case of a change of the valence electron state, and also for complex spd valence states.

Acknowledgments: The authors gratefully acknowledge Olga Degtyareva for her valuable discussion and comments. This work is supported by the Program "The Matter under High Pressure" of the Russian Academy of Sciences.

Author Contributions: Valentina Degtyareva conceived the project and wrote the paper; Nataliya Afonikova analyzed the results, prepared figures and tables.

Conflicts of Interest: The authors declare no conflict of interest.

\section{References}

1. Hume-Rothery, W. Researches on the nature, properties, and condition of formation of intermetallic compounds. J. Inst. Met. 1926, 35, 319-335.

2. Mott, N.F.; Jones, H. The Theory of the Properties of Metals and Alloys; Oxford University Press: London, UK, 1936.

3. Jones, H. The Theory of Brillouin Zones and Electron States in Crystals; North Holland Publ.: Amsterdam, The Netherlands, 1962.

4. Degtyareva, V.F.; Afonikova, N.S. Simple metal binary phases based on the body centered cubic structure: Electronic origin of distortions and superlattices. J. Phys. Chem. Solids 2013, 74, 18-24. [CrossRef]

5. Degtyareva, V.F.; Afonikova, N.S. Simple metal and binary alloy phases based on the $h c p$ structure: Electronic origin of distortions and superlattices. Solid State Sci. 2014, 37, 47-54. [CrossRef]

6. Degtyareva, V.F.; Afonikova, N.S. Complex structures in the Au-Cd alloy system: Hume-Rothery mechanism as origin. Solid State Sci. 2015, 49, 61-67. [CrossRef]

7. Lee, S.; Henderson, R.; Kaminsky, C.; Nelson, Z.; Nguyen, J.; Settje, N.F.; Schmidt, J.T.; Feng, J. Pseudo-fivefold diffraction symmetries in tetrahedral packing. Chem. Eur. J. 2013, 19, 10244-10270. [CrossRef] [PubMed]

8. Mizutani, U. Hume-Rothery Rules for Structurally Complex Alloy Phases; Taylor\& Francis: London, UK, 2010.

9. Berger, R.F.; Walters, P.L.; Lee, S.; Hoffmann, R. Connecting the chemical and physical viewpoints of what determines structure: From 1-D chains to gamma-brasses. Chem. Rev. 2011, 111, 4522-4545. [CrossRef] [PubMed]

10. Lee, S.; Leighton, C.; Bates, F.S. Sphericity and symmetry breaking in the formation of Frank-Kasper phases from one component materials. Proc. Natl. Acad. Sci. USA 2014, 111, 17723-17731. [CrossRef] [PubMed]

11. Feng, J.; Hoffmann, N.W. Ashcroft, Double-diamond NaAl via pressure: Understanding structure through Jones zone activation. J. Chem. Phys. 2010, 132, 114106. [CrossRef] [PubMed]

12. Degtyareva, V.F. Brillouin zone concept and crystal structures of sp metals under high pressure. High Press Res. 2003, 23, 253-257. [CrossRef]

13. Degtyareva, V.F. Simple metals at high pressures: The Fermi sphere-Brillouin zone interaction model. Physics-Uspekhi 2006, 49, 369-388. [CrossRef]

14. Degtyareva, V.F.; Degtyareva, O. Structure stability in the simple element sodium under pressure. New J. Phys. 2009, 11, 063037. [CrossRef]

15. Degtyareva, V.F. Potassium under pressure: Electronic origin of complex structures. Solid State Sci. 2014, 36, 62-72. [CrossRef]

16. Degtyareva, V.F. Structural simplicity and complexity of compressed calcium: Electronic origin. Acta Crystallogr. B 2014, 70, 423-428. [CrossRef] [PubMed] 
17. Degtyareva, V.F. Electronic Origin of the Orthorhombic Cmca Structure in Compressed Elements and Binary Alloys. Crystals 2013, 3, 419-430. [CrossRef]

18. Degtyareva, V.F.; Smirnova, I.S. BRIZ: A visualization program for Brillouin zone-Fermi sphere configuration. Z. Kristallogr. 2007, 222, 718-721. [CrossRef]

19. Villars, P.; Cenzual, K. (Eds.) Pauling File Binaries Edition; ASM International: Metal Park, OH, USA, 2002.

20. Pearson, W.B. The Crystal Chemistry and Physics of Metals and Alloys; Wileyl: New York, NY, USA, 1972.

21. Pearson, W.B. A Handbook of Lattice Spacings and Structures of Metals; Pergamon Press: New York, NY, USA, 1967; Volume 2.

22. Dubrovinsky, L.; Dubrovinskaia, N.; Crichton, W.A.; Mikhaylushkin, A.S.; Simak, S.I.; Abrikosov, I.A.; de Almeida, J.S.; Ahuja, R.; Luo, W.; Johansson, B. Noblest of All Metals Is Structurally Unstable at High Pressure. Phys. Rev. Lett. 2007, 98, 045503. [CrossRef] [PubMed]

23. McMahon, M.I.; Nelmes, R.J. High-pressure structures and phase transformations in elemental metals. Chem. Soc. Rev. 2006, 35, 943-963. [CrossRef] [PubMed]

24. Degtyareva, V.F.; Bdikin, I.K.; Porsch, F.; Novokhatskaya, N.I. The stability of the rhombohedral $\alpha-\mathrm{Hg}$ phase alloyed with Sn under high pressure up to 30 GPa. J. Phys. Condens. Matter 2003, 15, 7489-7500. [CrossRef]

25. Takemura, K.; Fujihisa, H.; Nakamoto, Y.; Nakano, S.; Ohishi, Y. Crystal Structure of the High-Pressure Phase of Mercury: A Novel Monoclinic Distortion of the Close-Packed Structure. J. Phys. Soc. Jpn. 2007, 76, 023601. [CrossRef]

26. Takemura, K.; Nakano, S.; Ohishi, Y.; Nakamotoand, Y.; Fujihisa, H. High-pressure structural study of solid mercury up to 200GPa. Mater. Res. Express 2015, 2, 016502. [CrossRef]

27. Biering, S.; Schwerdtfeger, P. High-pressure transitions in bulk mercury: A density functional study. Theor. Chem. Acc. 2011, 130, 455-462. [CrossRef]

28. Hanfland, M.; Syassen, K.; Christensen, N.E.; Novikov, D.L. New high-pressure phases of lithium. Nature 2000, 408, 174-178. [PubMed]

29. Ashcroft, N.W. Quantum-solid behavior and the electronic structure of the light alkali metals. Phys. Rev. B 1989, 39, 10552-10559. [CrossRef]

30. Schulte, O.; Nikolaenko, A.; Holzapfel, W.B. Pressure-Volume Relations For Zn, Cd, Ga, In and Tl at Room Temperature To $30 \mathrm{GPa}$ and Above. High Press. Res. 1991, 6, 169-182. [CrossRef]

31. Cazorla, C.; MacLeod, S.G.; Errandonea, D.; Munro, K.A.; McMahon, M.I.; Popescu, C. Thallium under extreme compression. J. Phys. Condens. Matter 2016, 28, 445401. [CrossRef] [PubMed]

32. Degtyareva, O.; Degtyareva, V.F.; Porsch, F.; Holzapfel, W.B. Face-centred cubic to tetragonal transitions in In alloys under high pressure. J. Phys. Condens. Matter 2001, 13, 7295-7303. [CrossRef]

33. Degtyareva, O.; McMahon, M.I.; Allan, D.R.; Nelmes, R.J. Structural Complexity in Gallium under High Pressure: Relation to Alkali Elements. Phys. Rev. Lett. 2004, 93, 205502. [CrossRef] [PubMed]

34. Sugimoto, T.; Akahama, Y.; Ichikawa, T.; Fujihisa, H.; Hirao, N.; Ohishi, Y. Bcc-fcc structure transition of Te. J. Phy. Conf. Ser. 2014, 500, 192018. [CrossRef]

35. Takemura, K.; Sato, K.; Fujihisa, H.; Onoda, M. Modulated structure of solid iodine during its molecular dissociation under high pressure. Nature 2003, 423, 971-974.

36. Fujii, Y.; Hase, K.; Hamaya, N.; Ohishi, Y.; Onodera, A.; Shimomura, O.; Takemura, K. Pressure-induced face-centered-cubic phase of monatomic metallic iodine. Phys. Rev. Lett. 1987, 58, 796-799. [CrossRef] [PubMed]

37. Reichlin, R.; McMahan, A.; Ross, M.; Martin, S.; Hu, J.; Hemley, R.; Mao, H.; Wu, Y. Optical, X-ray, and band-structure studies of iodine at pressures of several megabars. Phys. Rev. B 1994, 49, 3725-3731. [CrossRef]

38. Sato, H.; Toth, R.S. Fermi surface of Alloys. Phys. Rev. Lett. 1962, 8, 239-241. [CrossRef]

39. Sato, H.; Toth, R.S. Long-Period Superlattices in Alloys. II. Phys. Rev. 1962, 127, 469-484. [CrossRef]

40. Iwasaki, H.; Hirabayashi, M.; Fujiwara, K.; Watanabe, D.; Ogawa, S. Study on the Ordered Phases with Long Period in the Gold-Zinc Alloy System I. Survey of Crystal Structures and Calorimetric Measurement. J. Phys. Soc. Jpn. 1960, 15, 1771-1783. [CrossRef]

41. Terasaki, O. Study of the Incommensurate Two-Dimensional Antiphase Structure of $\mathrm{Au}^{3+} \mathrm{Zn}$ by High Voltage, High Resolution Electron Microscopy. J. Phys. Soc. Jpn. 1982, 51, 2159-2167. [CrossRef]

42. Iwasaki, H. Study on the Ordered Phases with Long Period in the Gold-Zinc Alloy System II. Structure Analysis of Au3Zn[R1], Au3Zn[R2] and Au ${ }^{3+}$ Zn. J. Phys. Soc. Jpn. 1962, 17, 1620-1633. [CrossRef] 
43. Betterton, J.O.; Hume Rothery, W. The Equilibrium diagram of the system copper-Gallium in the region 30-100 at. \% gallium. J. Inst. Met. 1952, 80, 459-468.

44. Heine, V.; Weaire, D. Pseudopotential Theory of Cohesion and Structure. Solid State Phys. 1970, $24,249-463$.

45. Smirnova, E.A.; Korzhavyi, P.A.; Vekilov, Y.K.; Johansson, B.; Abrikosov, I.A. Electronic topological transitions and phase stability in the fcc Al-Zn alloys. Eur. Phys. J. B 2002, 30, 57-66. [CrossRef]

46. Fujinaga, Y.; Tasaku, S. The aluminum-zinc phase diagram under high pressure. J. Alloys Compd. 1994, 209, 311-317. [CrossRef]

(C) 2017 by the authors; licensee MDPI, Basel, Switzerland. This article is an open access article distributed under the terms and conditions of the Creative Commons Attribution (CC BY) license (http:/ / creativecommons.org/licenses/by/4.0/). 